\title{
Research of Operation of Predictive Controllers of Distributed Generation Plant in Power Supply System with Energy Storage
}

\author{
Yuri N. Bulatov*a, \\ Andrey V. Kryukov ${ }^{\mathrm{b}, \mathrm{c}}$ and Konstantin V. Suslov ${ }^{\mathrm{c}}$ \\ ${ }^{a}$ Bratsk State University \\ Bratsk, Russian Federation \\ ${ }^{\mathrm{b}}$ Irkutsk State Transport University \\ Irkutsk, Russian Federation \\ 'Irkutsk National Research Technical University \\ Irkutsk, Russian Federation
}

Received 12.04.2021, received in revised form 11.05.2021, accepted 21.06.2021

\begin{abstract}
The article discusses the power supply system of an industrial enterprise, which included a turbine generator plant operating on the basis of a synchronous generator equipped with predictive voltage and rotor speed controllers, as well as a high-power electric energy storage device. A description of the models of this plant, predictive controllers and energy storage, as well as the results of modeling when the system goes into an isolated mode of operation are given. Simulation was performed in MATLAB environment using Simulink and SimPowerSystems packages. The purpose of the work was to study the behavior of the proposed predictive controllers during the transition of the power supply system to the island (isolated) mode. Based on the results of computer simulation, it was concluded that the use of predictive controllers improves the damping properties of the system. The use of an energy storage device that is automatically connected to the network when the voltage drops, allows to reduce the overvoltage at the terminals of the generator during its unloading, as well as to reduce the required mechanical power on the turbine shaft in comparison with a permanently connected device. Predictive controllers can be recommended to increase the stability of distributed generation plants when switching to an isolated mode. It is advisable to conduct further research in the direction of creating algorithms for coordinated operation of controllers.
\end{abstract}

Keywords: distributed generation plants, power supply systems, energy storage, isolated mode, asynchronous load, automatic voltage controller, automatic speed controller, simulation.

Citation: Bulatov Yu.N., Kryukov A. V., Suslov K. V. Research of operation of predictive controllers of distributed generation plant in power supply system with energy storage, J. Sib. Fed. Univ. Eng. \& Technol., 2021, 14(4), 448-458. DOI: 10.17516/1999494X-0325

(c) Siberian Federal University. All rights reserved

This work is licensed under a Creative Commons Attribution-Non Commercial 4.0 International License (CC BY-NC 4.0).

* Corresponding author E-mail address: bulatovyura@yandex.ru 


\title{
Исследование работы прогностических
}

\section{регуляторов установки распределенной генерации \\ в системе электроснабжения}

\section{с мощным накопителем электроэнергии}

\author{
Ю. Н. Булатов ${ }^{\text {a }}$ А. В. Крюков ${ }^{\tilde{\sigma}, \text { в }, \text { К. В. Суслов }}{ }^{\text {в }}$ \\ ${ }^{a}$ Братский государственный университет \\ Российская Федерация, Братск \\ ${ }^{\sigma}$ Иркутский государственный университет путей сообщения \\ Российская Федерачии, Иркутск \\ ${ }^{8}$ Иркутский национальный \\ исследовательский технический университет \\ Российская Федерация, Иркутск
}

\begin{abstract}
Аннотация. В статье рассматривается система электроснабжения промышленного предприятия, в состав которой входила турбогенераторная установка, работающая на основе синхронного генератора, оснащенного прогностическими регуляторами напряжения и скорости вращения ротора, а также накопитель электроэнергии большой мощности. Приведено описание моделей этой установки, прогностических регуляторов и накопителя энергии, а также результаты моделирования при переходе системы в изолированный режим работы. Моделирование выполнено в среде MATLAB с использованием пакетов Simulink и SimPowerSystems. Цель работы состояла в исследовании поведения предлагаемых прогностических регуляторов при переходе системы электроснабжения в островной (изолированный) режим. По результатам компьютерного моделирования сделан вывод, что использование прогностических регуляторов позволяет улучшить демпфирующие свойства системы. Применение накопителя энергии, автоматически подключаемого к сети при снижении напряжения, позволяет уменьшить перенапряжение на зажимах генератора при его разгрузке, а также снизить необходимую механическую мощность на валу турбины в сравнении с постоянно подключенным устройством. Прогностические регуляторы могут быть рекомендованы для повышения устойчивости работы установок распределенной генерации при переходе в изолированный режим. Целесообразно проведение дальнейших исследований в направлении создания алгоритмов согласованной работы регуляторов.
\end{abstract}

Ключевые слова: установки распределенной генерации, системы электроснабжения, накопители электроэнергии, изолированный режим, асинхронная нагрузка, автоматический регулятор возбуждения, автоматический регулятор скорости, моделирование.

Цитирование: Булатов, Ю.Н. Исследование работы прогностических регуляторов установки распределенной генерации в системе электроснабжения с мощным накопителем электроэнергии / Ю.Н. Булатов, А. В. Крюков, К. В. Суслов // Журн. Сиб. федер. ун-та. Техника и технологии, 2021, 14(4). С. 448-458. DOI: 10.17516/1999-494X-0325

\section{Введение}

При развитии и модернизации систем электроснабжения (СЭС) в современных условиях целесообразно применять установки распределенной генерации (РГ) [1], позволяющие повысить надежность электроснабжения потребителей и уменьшить потери при передаче электроэнергии [2]. Кроме этого, использование установок РГ оказывает положительное влияние на качество электроэнергии в СЭС $[3,4]$.

$$
-449-
$$


В режиме параллельной работы установок РГ и электроэнергетической системы (ЭЭС) большой мощности повышается надежность электроснабжения и эффективность стабилизации напряжения и частоты при различных возмущениях. В то же время такой режим приводит к увеличению токов короткого замыкания, усложнению устройств релейной защиты и задач управления режимами. В аварийных ситуациях целесообразно использовать островной (изолированный) режим, когда установки РГ выделяются в кластеры для питания части ответственных потребителей $[5,6]$. С целью повышения надежности функционирования СЭС необходимо решить ряд задач, в число которых входит оптимальное управление установками РГ при переходе в изолированный режим работы [7]; при этом необходимо учитывать типы используемых установок РГ и их генераторов, ограничения по максимальной мощности потребителей, характер нагрузки, а также влияние на генерирующие установки резкого повышения или понижения нагрузки.

Установки РГ, работающие на основе синхронных турбо- и гидрогенераторов, позволяют получить достаточную мощность для питания промышленных потребителей. Повысить устойчивость работы генераторов позволяют автоматические регуляторы возбуждения (AРВ) и скорости (АРС) вращения ротора. Задачи построения и настройки регуляторов установок РГ, работающих в СЭС при различных режимах, могут быть решены с использованием накопителей электроэнергии [8] и интеллектуальных технологий управления $[9,10]$.

Проведенные исследования показывают, что эффективным способом управления синхронными генераторами является использование нечетких регуляторов $[11,12]$ и прогностических алгоритмов [13, 14], позволяющих создавать адаптивные системы. Однако для практического применения таких систем требуются трудоемкие исследования на сложных моделях с учетом большого числа возможных режимов работы для определения их влияния на параметры регулирования и показатели качества процесса управления.

Целью данной работы является исследование поведения предлагаемых прогностических АРВ и АРС при переходе СЭС с мощной асинхронной нагрузкой в островной (изолированный) режим. Исследования проводили для СЭС промышленного предприятия с турбогенераторной установкой (ТГУ) и накопителем электроэнергии (НЭ) большой мощности. Моделирование выполняли в системе MATLAB с использованием пакетов Simulink и SimPowerSystems. Ниже представлено описание используемой модели СЭС с установкой РГ и НЭ, а также результатов моделирования.

\section{Описание используемой модели СЭС с установкой РГ и НЭ}

Схема исследуемой СЭС промышленного предприятия, показанная на рис. 1 , имела связь с ЭЭС через два трансформатора (Т-1 и Т-2) 110/10 кВ мощностью по 6300 кВ·А каждый. Основными потребителями электроэнергии в рассматриваемой СЭС являются асинхронные двигатели (АД): два высоковольтных АД мощностью по 670 кВт каждый, а также большое количество низковольтных АД, учитываемых в модели в виде эквивалентных блоков мощностью 930 и 1485 кВт соответственно, с трансформаторами и кабельными линиями, получающими питание от разных секций шин (рис. 1). В состав СЭС входит ТГУ мощностью 3125 кВ×А и НЭ большой мощности. Схема имитационной модели СЭС в MATLAB представлена на рис. 2. 


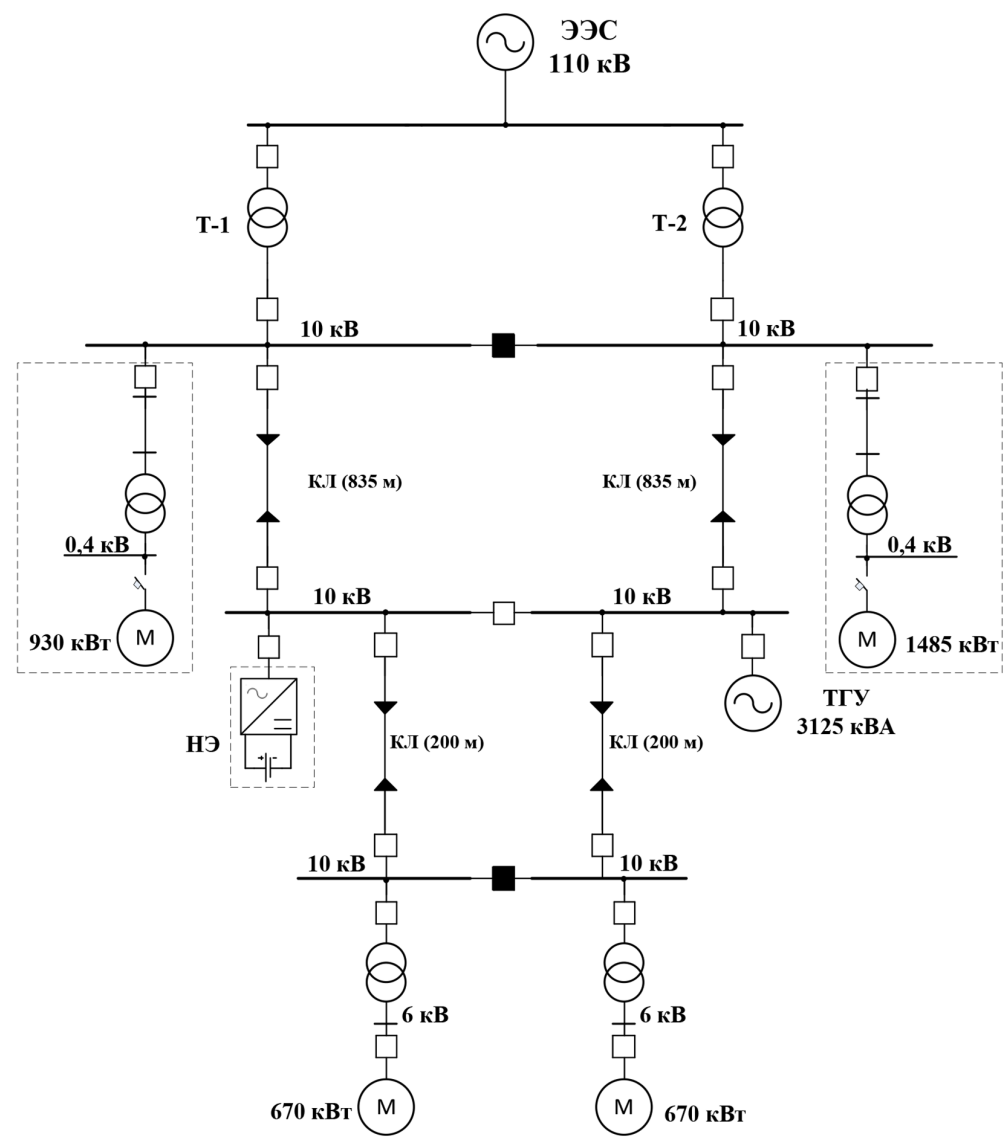

Рис. 1. Схема исследуемой СЭС промышленного предприятия: КЛ - кабельная линия

Fig. 1. Scheme of the investigated power supply system (PSS) of an industrial enterprise: CL - cable line

Синхронный генератор ТГУ (блок ТGP на рис. 2) моделировался блоком пакета SymPowerSystems - Synchronous Machine. Модель используемой паровой турбины ТГУ описывалась следующим дифференциальным уравнением:

$$
T_{T} \cdot \frac{d P_{T}}{d t}+P_{T}=\mu
$$

где $P_{T}$ - мощность турбины; $\mu$ - открытие регулирующего органа; $T_{T}$ - постоянная времени турбины (принималась из практических соображений равной 0,2 с [14]).

Система возбуждения генератора ТГУ моделировалась апериодическим звеном первого порядка с передаточной функцией вида [15]

$$
\frac{1}{0,025 s+1},
$$

а также последовательно соединенным с ним усилителем с передаточной функцией

$$
\frac{1}{0,001 s+1}
$$

где s - оператор Лапласа. 
Для повышения устойчивости синхронного генератора ТГУ в модели применялись АРВ и АРС с использованием прогностических звеньев или без них. В качестве регулятора возбуждения синхронного генератора ТГУ предлагалось использовать прогностический АРВ, структурная схема Simulink-модели которого представлена на рис. 3. Схема используемой Simulink-модели прогностического АРС показана на рис. 4 [14]. Из схем, приведенных на рис. 3 и 4, видно, что на выходе регуляторов подключается линейное прогнозирующее звено с передаточной функцией $T_{p} s+1$ [16] и последовательно соединенным с ним электронным усилителем с передаточной функцией $\frac{K_{a}}{T_{a} s+1}$. При моделировании принимались следующие числовые значения параметров: $K_{a}=1 ; T_{a}=0,001 \mathrm{c}$.

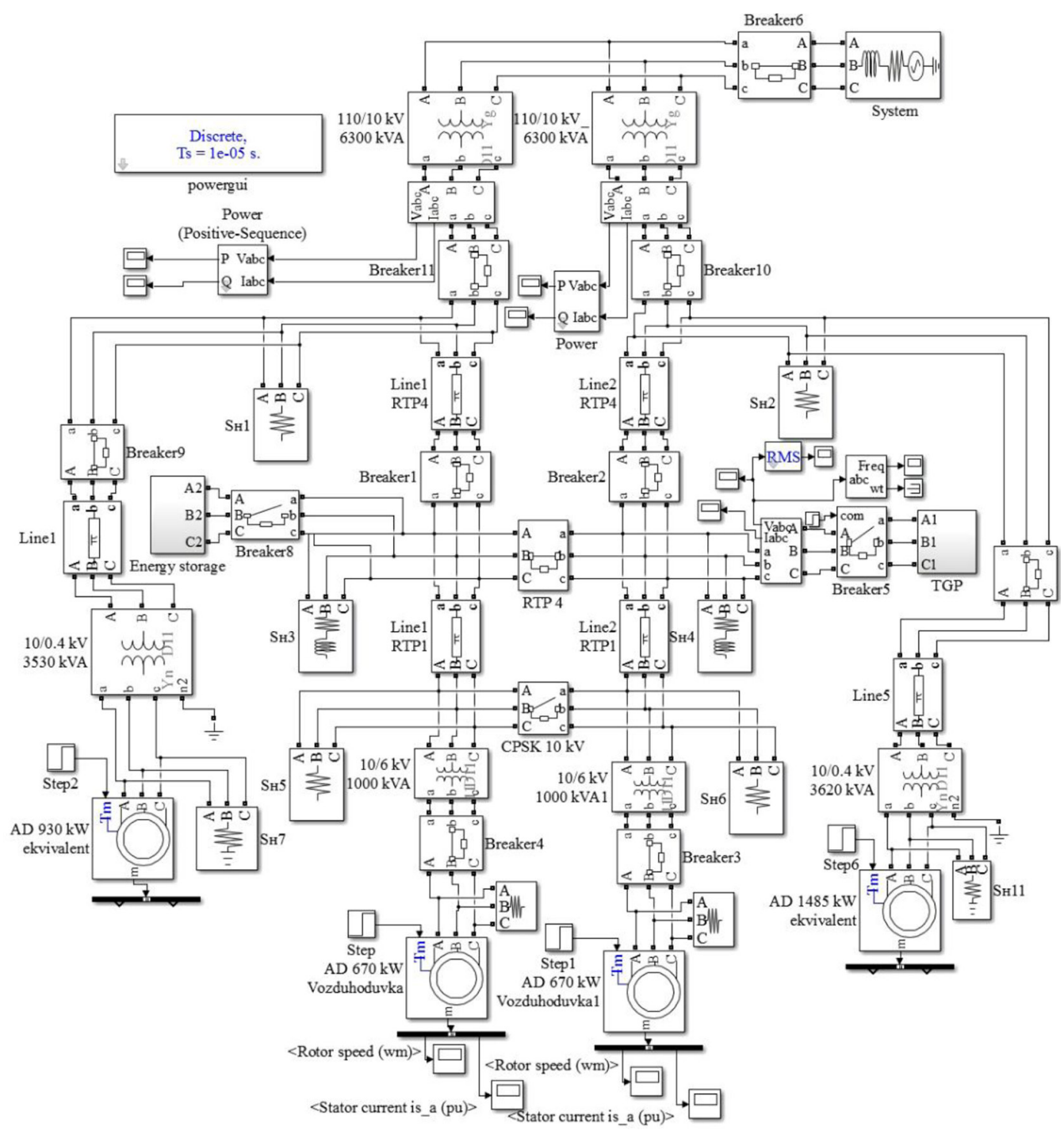

Рис. 2. Схема имитационной модели в MATLAB

Fig. 2. Simulation model diagram in MATLAB 


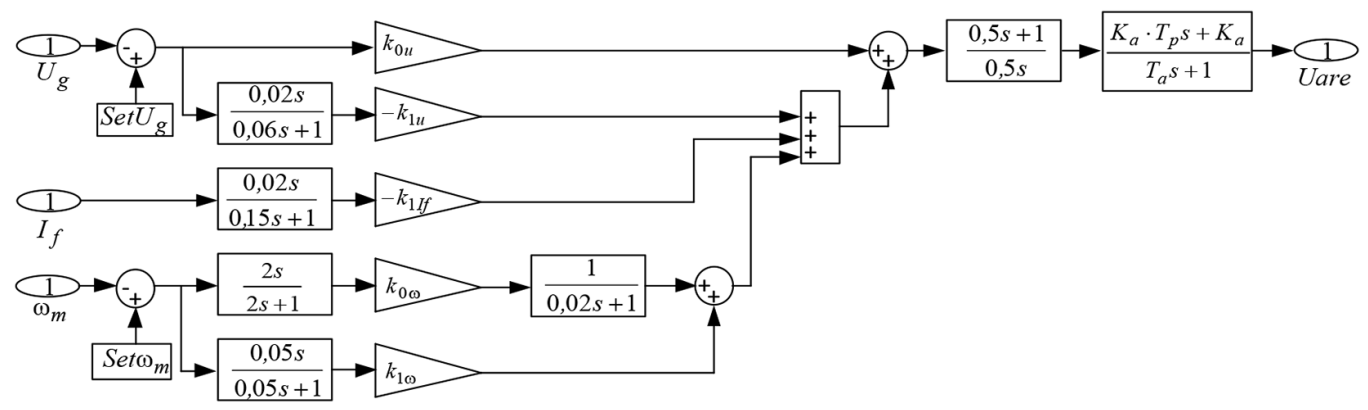

Рис. 3. Схема Simulink-модели прогностического APB: $U_{g}$ - текущее значение напряжения генератора; $\operatorname{Set} U_{g}$ - заданное значение напряжения генератора; $I_{f}$ - ток возбуждения генератора; $\omega_{m}$ - текущее значение частоты вращения ротора генератора; Set $\omega_{m}$ - заданное значение частоты вращения ротора генератора; $k_{0 u}, k_{1 u}, k_{1 I f}, k_{0 \omega}, k_{1 \omega}-$ коэффициенты настройки APB; $T_{p}$ - постоянная времени прогнозирующего звена

Fig. 3. Simulink diagram of predictive AVC model: $U_{g}$ - an instantaneous value of generator voltage; $\operatorname{Set} U_{g}-$ a set value of generator voltage; $I_{f}$ - generator excitation current; $\omega_{m}$-a generator rotor speed instantaneous value; Set $\omega_{m}$ - a generator rotor speed set value; $k_{0 u}, k_{1 u}, k_{1 I f}, k_{0 \omega}, k_{1 \omega}$ - tuning coefficients of AVC; $T_{p}-$ the prognostic link time constant

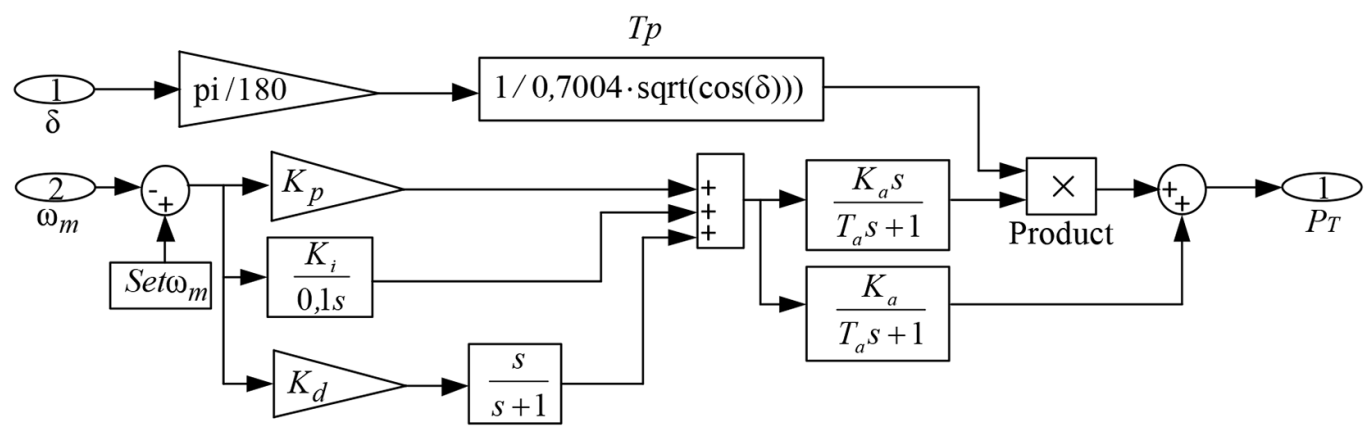

Рис. 4. Схема Simulink-модели прогностического APC: $K_{p}, K_{i}, K_{d}$ - коэффициенты настройки АРC

Fig. 4. Simulink diagram of predictive ASC model: $K_{p}, K_{i}, K_{d}$-tuning coefficients of ASC

Коэффициенты настройки АРВ и АРС определялись из практических соображений и принимались одинаковыми для классических ПИД регуляторов и устройств с прогностическим звеном. Постоянная времени прогноза для АРС определялась автоматически [14] и изменялась в зависимости от угла нагрузки генератора $\delta$ согласно следующей функции:

$$
T_{p}^{A P C}=\frac{1}{f_{p}(\delta)}=\frac{1,428}{\sqrt{\cos \delta}}
$$

Постоянная времени прогнозирующего звена для АРВ $T_{p}^{A P B}$ определялась в соответствии с методикой, описанной в [14], и принималась равной 0,125 с. Следует отметить, что используемая методика является универсальной и может применяться в схемах с любым количеством генераторов и потребителей электроэнергии.

В исследуемой СЭС предполагалось использование литий-ионных аккумуляторных батарей в связи с их преимуществами перед другими типами НЭ [17]. В качестве модели литийионных аккумуляторных батарей использовался блок Battery пакета SimPowerSystems системы

$$
-453-
$$


MATLAB, который подключался к трехфазной сети переменного тока через инвертор и LC фильтр. Зарядка НЭ, мощность которого при моделировании составляла 3 МВт, может осуществляться от ЭЭС или от ТГУ в часы минимальных нагрузок.

\section{Результаты моделирования}

Исследования проводили при переходе СЭС в изолированный режим работы при следующей технической оснащенности установок РГ:

- использование ТГУ с прогностическими АРВ и АРС [14] или с классическими регуляторами;

- использование в СЭС НЭ большой мощности, постоянно подключенного или подключаемого автоматически при снижении напряжения.

Начальная загрузка генератора ТГУ составляла 80 \%, и при переходе в островной режим работы генератор оказывался перегруженным. Длительное время такой режим не может существовать, поэтому для компенсации дефицита генерирующей мощности в СЭС использовался НЭ, мощность и емкость которого была достаточна для длительного питания потребителей. Результаты моделирования перехода СЭС в изолированный режим работы в виде временных зависимостей мощности на валу турбины ТГУ, скорости вращения ротора и напряжения генератора показаны на рис. 5 .

На рис. 6 изображены временные зависимости частоты в СЭС в указанном режиме работы. По представленным на рис. 5 и 6 временным зависимостям видно, что использование в СЭС НЭ большой мощности позволяет разгрузить генератор ТГУ и эффективнее стабилизировать частоту в сети при переходе СЭС в изолированный режим. Однако при этом наблюдается увеличение перерегулирования, колебательности и времени переходного процесса для скорости вращения ротора и напряжения генератора ТГУ.

Можно отметить, что использование прогностических АРВ и АРС позволяет улучшить демпферные свойства системы без применения процедур оптимизации настроек регуляторов: снижается величина перерегулирования, колебательность и время переходного процесса для скорости вращения ротора генератора, мощности на валу турбины и частоты напряжения в сети. В рассматриваемом режиме на характер изменения амплитуд напряжения генератора ТГУ прогностический АРВ влияния практически не оказывает.

Дополнительно было выполнено моделирование перехода СЭС в изолированный режим работы при отключенном НЭ, который подключался автоматически при снижении напряжения. На рис. 7 показаны результаты моделирования в виде параметров ТГУ и частоты в сети СЭС.

Сравнительный анализ результатов моделирования перехода СЭС в изолированный режим работы позволяет сделать вывод об эффективности применения НЭ большой мощности для повышения надежности электроснабжения потребителей и недопущения режима перегрузки используемой установки РГ. По сравнению с ситуацией, когда НЭ отключен, наблюдаются несколько большие отклонения и колебания напряжения и скорости вращения ротора генератора ТГУ, что может быть сведено к минимуму благодаря использованию прогностических АРВ и АРС. 

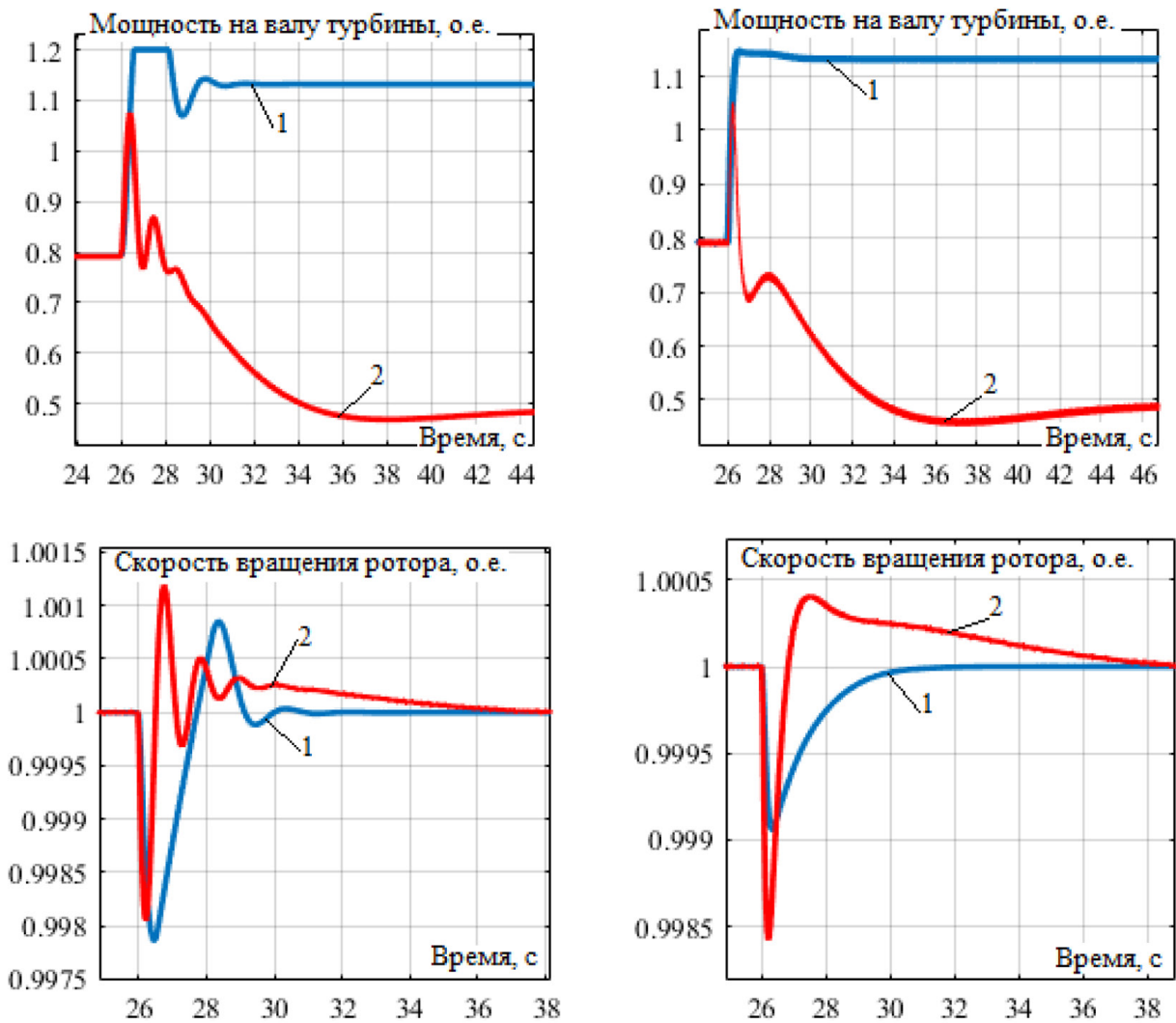

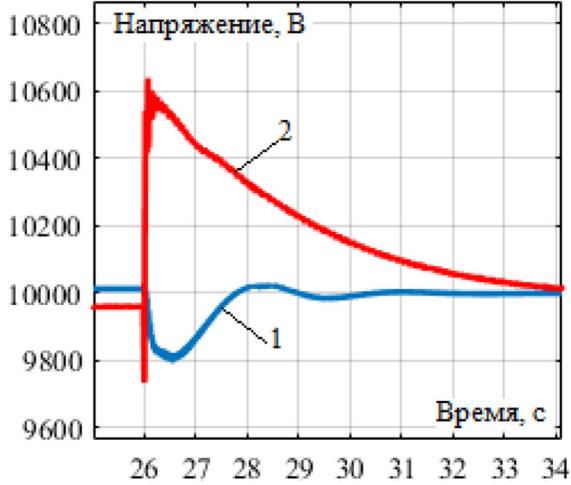

a)

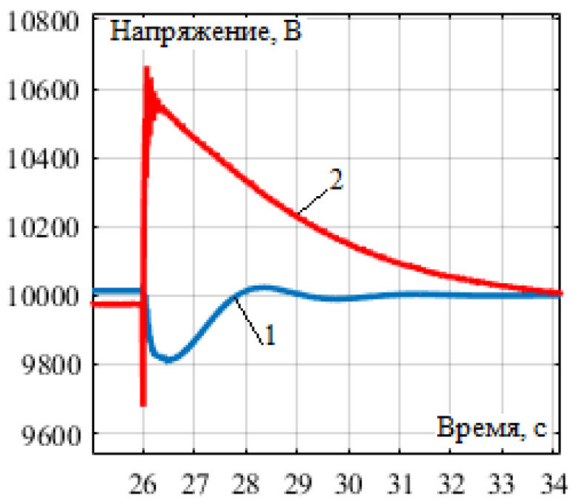

б)

Рис. 5. Параметры ТГУ при переходе в островной (изолированный) режим работы СЭС: a использовались классические АРВ и АРС; б - использовались прогностические АРВ и АРС; 1 - НЭ отключен; 2 - НЭ всегда включен

Fig. 5. Parameters of the TGP during the transition to the island (isolated) operation mode of the PSS: a - classic AVC and ASC were used; 6 - predictive AVC and ASC were used; 1 - energy storage (ES) is disabled; 2 - ES is always on 


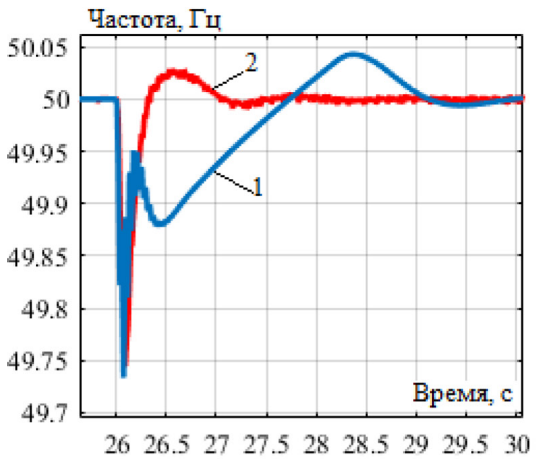

a)

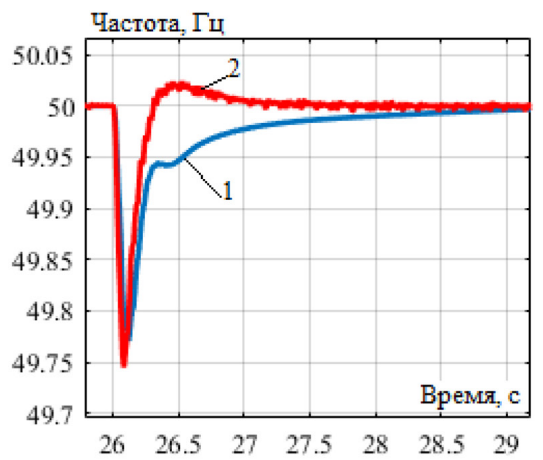

б)

Рис. 6. Частота в сети при переходе СЭС в островной (изолированный) режим работы: А использовались классические АРВ и АРС; б - использовались прогностические АРВ и АРС; 1 - НЭ отключен; 2 - НЭ всегда включен

Fig. 6. The frequency in the mains during the PSS transition to the island (isolated) operation mode: $\mathrm{a}-$ classic AVC and ASC were used; 6 - predictive AVC and ASC were used; 1 - ES is disabled; 2 - ES is always on

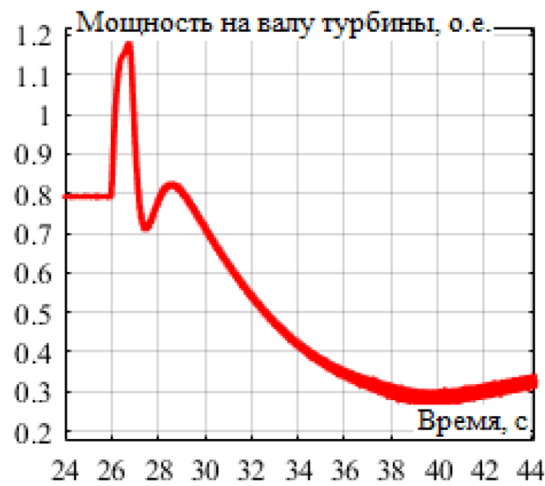

a)

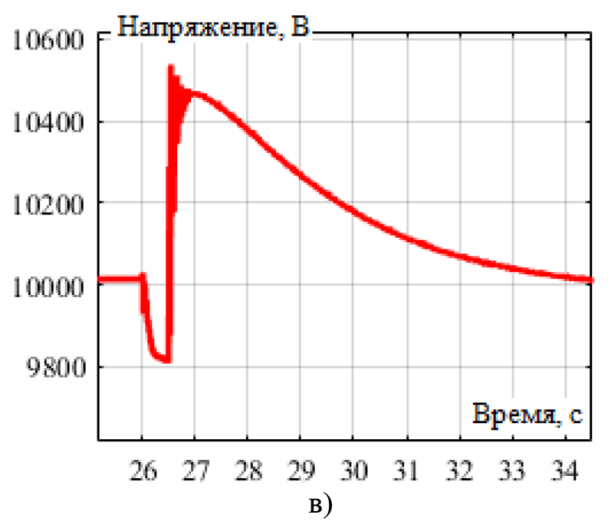

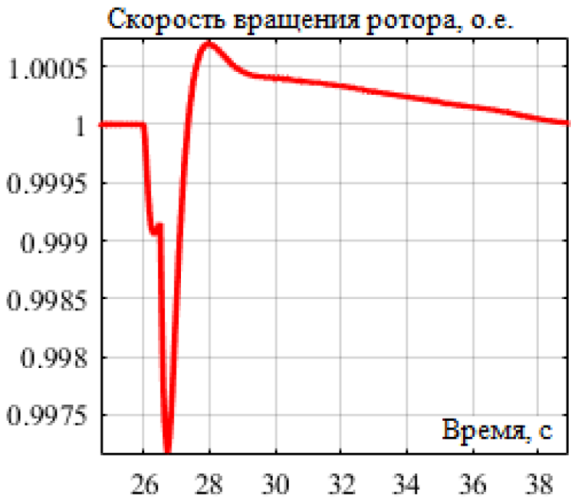

б)

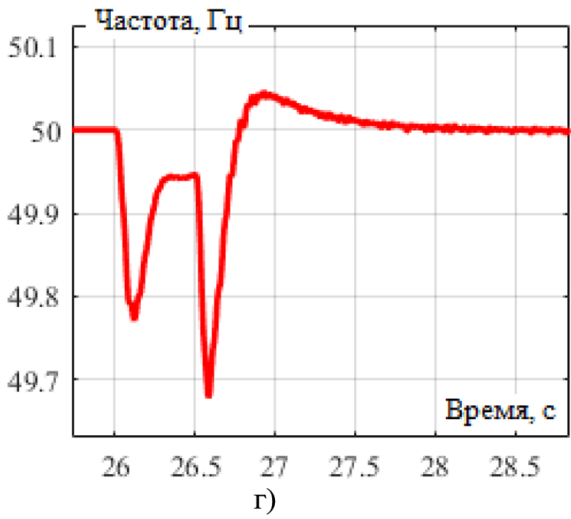

Рис. 7. Параметры ТГУ с прогностическими АРВ и АРС (a, б, в) и частота в сети (г) при переходе СЭС в изолированный режим работы: НЭ подключался автоматически при снижении напряжения

Fig. 7. TGP parameters with predictive AVC and ASC (а, б, в) and frequency in the mains (г) when the PSS transition to an isolated operation mode: ES is connected automatically when the voltage drops 


\section{Заключение}

По результатам компьютерного моделирования можно сделать следующие выводы:

1. Использование накопителей энергии большой мощности позволяет разгрузить генератор ТГУ и не отключать важных потребителей, что особенно актуально для СЭС с дефицитом генерирующих установок.

2. Применение накопителей во всех рассматриваемых режимах дает возможность более эффективно стабилизировать частоту в сети, однако при этом наблюдается увеличение перерегулирования, колебательности и времени переходного процесса для скорости вращения ротора и напряжения генератора ТГУ. Возникающее перенапряжение на зажимах генератора при переходе в островной режим работы объясняется резким сбросом нагрузки ТГУ при перераспределении питания потребителей.

3. Использование прогностического АРС генератора ТГУ даже без применения процедур оптимизации настроек регуляторов позволяет снизить величину перерегулирования, колебательность и время переходного процесса для скорости вращения ротора генератора, мощности на валу турбины и частоты напряжения в сети. На изменение амплитуд напряжения генератора ТГУ в рассматриваемом режиме прогностический АРВ влияния практически не оказывает.

4. Использование НЭ, автоматически подключаемого к сети СЭС при снижении напряжения, дает возможность несколько уменьшить перенапряжение на зажимах генератора при его разгрузке, а также больше снизить необходимую механическую мощность на валу турбины ТГУ в сравнении с постоянно подключенным НЭ.

5. Предлагаемые прогностические регуляторы синхронных генераторов могут быть рекомендованы для повышения устойчивости установок РГ при переходе в изолированный режим. Целесообразно проведение дальнейших исследований в направлении создания алгоритмов согласованной работы регуляторов установки РГ и накопителя электроэнергии.

\section{Благодарности / Acknowledgements}

Работа выполнена в рамках государственного задания Министерства науки и высшего образования Российской Федерации (проект № 0667-2020-0039).

The work was carried out within the framework of the state assignment of the Ministry of Science and Higher Education of the Russian Federation (project No. 0667-2020-0039).

\section{Список литературы / References}

[1] Ackermann T., Anderson G., and Söder L. Distributed generation: a definition, Electric Power Systems Research, 2001, 57, 195-204.

[2] Rugthaicharoencheep N., Auchariyamet S. Technical and Economic Impacts of Distributed Generation on Distribution System, International Journal of Electrical, Computer, Energetic, Electronic and Communication Engineering, 2012, 6(4), 385-389.

[3] Sikorski T., Rezmer J. Distributed Generation and Its Impact on Power Quality in LowVoltage Distribution Networks, Power Quality Issues in Distributed Generation, Dr. Jaroslaw Luszcz (Ed.), InTech, 2015, DOI: 10.5772/61172.

[4] Hariri A., Faruque M. O. Impacts of distributed generation on power quality, North American Power Symposium (NAPS), 2014, 1-6.

$$
-457-
$$


[5] Martinez-Cid R., O'Neill-Carrillo E. Sustainable microgrids for isolated systems, Transmission and Distribution Conference and Exposition, 2010. IEEE PES New Orleans, LA, USA 19-22 April 2010

[6] Saleh M.S., Althaibani A., Esa Y., and Mhandi Y. Impact of clustering microgrids on their stability and resilience during blackouts, Proc. on Int. Conf. on Smart Grid and Clean Energy Technologies, 2015, 195-200.

[7] Arai J., Yamazaki S., Ishikawa M., Ito T. Study on a new power control of distributed generation in an isolated microgrid, Power \&Energy Society General Meeting, 2009. PES '09. IEEE26-30 July 2009. Calgary Canada

[8] Lombardi P., Styczynski Z. A., Sokolnikova T., Suslov K. Use of energy storage in Isolated Micro Grids, Power Systems Computation Conference (PSCC), IEEE Conference Publications, 2014, 1-6. DOI: 10.1109/PSCC.2014.7038361.

[9] Magdi S. Mahmoud, Fouad M. AL-Sunni, Control and Optimization of Distributed Generation Systems, Cham: Springer International Publishing: Imprint: Springer, 2015. 578 p.

[10] Wang R., Wang P., Xiao G. Intelligent Microgrid Management and EV Control Under Uncertainties in Smart Grid, Springer, 2018. 218 p.

[11] Voropai N.I., Etingov P. V. Two-Stage Adaptive Fuzzy PSS Application to Power Systems, Proc. of International Conference on Electrical Engineering ICEE'2001, July 22-26, Xi'an, China, 2001, 1, 314-318.

[12] Kryukov A. V., Kargapol'cev S.K., Bulatov Yu.N., Skrypnik O. N., Kuznetsov B. F. Intelligent control of the regulators adjustment of the distributed generation installation, Far East Journal of Electronics and Communications, 2017, 17(5), 1127-1140.

[13] Camacho E.F., Bordons C. Model Predictive Control, 2nd edition Springer, 2007. 405 p.

[14] Bulatov Y.N., Kryukov A. V., Nguen V.H. Automatic Prognostic Regulators of Distributed Generators, Int. Multi-Conf. on Industrial Engineering and Modern Technologies, 2018, 1-4. DOI: 10.1109/FarEastCon.2018.8602718

[15] Anderson P.M., Fouad A.A. Power System Control and Stability, Second Edition. IEEE Press, 2003. 688 p.

[16]Pikina G. A.,Pashchenko F. F.,Pashchenko A.F. Synthesis, ResearchandComparativeAnalysis of Predictive Control Algorithms, 2020 International Multi-Conference on Industrial Engineering and Modern Technologies (FarEastCon), 2020, 1-5. DOI: 10.1109/FarEastCon50210.2020.9271646

[17] Yoshio Nishi Lithium ion secondary batteries; past 10 years and the future, Journal of Power Sources, 2001, 100, 101-106. 